PROCEEDINGS OF THE

AMERICAN MATHEMATICAL SOCIETY

Volume 135, Number 12, December 2007, Pages 4019-4022

S 0002-9939(07)08695-9

Article electronically published on September 5, 2007

\title{
A CONTINUUM WHOSE HYPERSPACE OF SUBCONTINUA IS NOT ITS CONTINUOUS IMAGE
}

\author{
ALEJANDRO ILLANES \\ (Communicated by Alexander N. Dranishnikov)
}

\begin{abstract}
We construct a metric continuum $X$ such that the hyperspace of subcontinua, $C(X)$, of $X$ is not a continuous image of $X$. This answers a question by I. Krzemińska and J. R. Prajs.
\end{abstract}

\section{INTRODUCTION}

A continuum is a nonempty, nondegenerate compact connected metric space. Given a continuum $X$, we consider the following hyperspaces of $X$ :

$2^{X}=\{A \subset X: A$ is closed and nonempty $\}, C(X)=\left\{A \in 2^{X}: A\right.$ is connected $\}$.

Both are endowed with the Hausdorff metric $H$.

The problem of determining conditions for the existence of onto mappings between $X, C(X)$ and $2^{X}$ has been considered in [1], 2] and [3. In [2, 3.6] it was proved that there is always a mapping from $2^{X}$ onto $C(X)$. In [2, 3.5], it was also proved that there is a mapping from $X$ onto $2^{X}$ if and only if (a) $X$ is locally connected or (b) $X$ contains an open set with uncountably many components. Thus, if $X$ satisfies (a) or (b), then $C(X)$ is a continuous image of $X$. For a more detailed discussion on this topic see Chapter IV of [3. In [1, Question 2], I. Krzemińska and J. R. Prajs asked if there exists a continuum $X$ with no continuous surjection from $X$ onto $C(X)$ (this question also was posted on the Open Problems on Continuum Theory website of J. R. Prajs and W. J. Charatonik). Here, we answer this question in the negative by constructing a continuum $X$ such that $C(X)$ is not a continuous image of $X$. The related questions are (1) Is $C(C(X))$ a continuous image of $C(X)$ for each continuum $X$ ? ([1, Question 3]) and (2) If $Y$ is a continuous image of $X$, is $C(Y)$ a continuous image of $C(X)$ ? ([1, Question 1]). These questions remain open.

\section{The eXAmple}

Let $Z$ be the $\sin \left(\frac{1}{x}\right)$-continuum defined as the closure in the plane of the graph of the function $\sin \left(\frac{1}{x}\right)$ with the interval $(0,1]$ as its domain. Then $Z$ is the union of the limit arc $J=\{0\} \times[-1,1]$ and the ray $T=Z-J$. Consider the continuum $Y$ obtained by identifying the points $(0,-1)$ and $(0,1)$ in $Z$ and let $h: Z \rightarrow Y$ be the quotient map. Then $Y$ is the union of the simple closed curve $C=h(J)$ and

Received by the editors April 6, 2005 and, in revised form, March 3, 2006.

2000 Mathematics Subject Classification. Primary 54B20.

Key words and phrases. Continuous images, continuum, hyperspaces. 
the ray $h(T)$. The continuum $Y$ constructed in this way is going to be called a packman having its limit circle $C$, its ray $h(T)$ and its peak point $h((0,1))$.

Consider the circle $S=\left\{(x, y, 0) \in E^{3}: x^{2}+y^{2}=1\right\}$ in the Euclidean space $E^{3}$. Fix a sequence $\left\{z_{n}\right\}_{n=1}^{\infty}$ in $S$ and a point $z_{0} \in S$ such that $\lim z_{n}=z_{0}$ and the points $z_{0}, z_{1}, z_{2}, \ldots$ are pairwise different.

Consider a sequence of packman continua $\left\{Y_{n}\right\}_{n=1}^{\infty}$ in $E^{3}$ with the following properties:

(a) $S$ is the limit circle of $Y_{n}$ for each $n \in \mathbb{N}$,

(b) $z_{n}$ is the peak point of $Y_{n}$ for each $n \in \mathbb{N}$,

(c) the rays $R_{1}=Y_{1}-S, R_{2}=Y_{2}-S, \ldots$ are pairwise disjoint,

(d) $\lim Y_{n}=S$.

The continuum $X$ is then defined as $X=Y_{1} \cup Y_{2} \cup \ldots$. We can also assume that $X$ satisfies the following property:

(e) given a path $\alpha:[0,1] \rightarrow S$ and $\lambda>0$, there exists $\delta>0$ such that, if $z_{n} \notin \alpha([0,1]), q \in R_{n}$ and $\|\alpha(0)-q\|<\delta$, then there exists a path $\beta:[0,1] \rightarrow R_{n}$ such that $\beta(0)=q$ and $\|\alpha(t)-\beta(t)\|<\lambda$ for each $t \in[0,1]$.

Observe that $X$ satisfies the following properties:

(f) if $J$ is an arc in $S, z_{n} \in J$ and $z_{n}$ is not an end point of $J$, then $J \notin$ $\operatorname{cl}_{C(X)}\left(C\left(R_{n}\right)\right)$, where $C\left(R_{n}\right)=\left\{A \in C(X): A \subset R_{n}\right\}$,

(g) if $J$ is an arc in $S-\left\{z_{n}\right\}$, then $J \in \operatorname{cl}_{C(X)}\left(C\left(R_{n}\right)\right)$,

(h) $S$ is a terminal subcontinuum of $X$, that is, if $A \in C(X)$ and $A \cap S \neq \emptyset$, then $A \subset S$ or $S \subset A$.

We are going to prove that there is no continuous map from $X$ onto $C(X)$. By way of contradiction, suppose that there exists a continuous onto map $f: X \rightarrow$ $C(X)$.

Given $n \in \mathbb{N}$ and a continuum $J \subset S-\left\{z_{n}\right\}$, by property (g), there exists a sequence of continua $\left\{J_{m}\right\}_{m=1}^{\infty}$ such that $J_{m} \subset R_{n}$ for each $m \in \mathbb{N}$ and $\lim J_{m}=J$. Since $f$ is onto, for each $m \in \mathbb{N}$, there exists $p_{m} \in X$ such that $f\left(p_{m}\right)=J_{m}$. Since $X$ is compact, we can assume that $\lim p_{m}=p_{0}$, for some $p_{0} \in X$. By continuity of $f, f\left(p_{0}\right)=J$. We need to prove the following.

Claim 1. If $\left\{B_{k}\right\}_{k=1}^{\infty}$ is a sequence in $C(X)$ such that $p_{0} \in B_{k}$ for each $k \in \mathbb{N}$ and $\lim B_{k}=\left\{p_{0}\right\}$, then there exists $k \in \mathbb{N}$ such that $B_{k} \cap\left\{p_{1}, p_{2}, \ldots\right\}=\emptyset$.

In order to prove Claim 1, for each $k \in \mathbb{N}$, let $C_{k}=\bigcup\left\{f(p): p \in B_{k}\right\}$. It is easy to show that (see [3, Lemmas 1.43 and 1.48]) $J \subset C_{k} \in C(X)$ for each $k \in \mathbb{N}$ and $\lim C_{k}=J$. Since $z_{n} \notin J$, there exists $k \in \mathbb{N}$ such that $z_{n} \notin C_{k}$. By property (h), $C_{k} \subset S$. Thus $C_{k} \cap R_{n}=\emptyset$ and $C_{k}$ does not contain any set $J_{m}$. Hence, $B_{k} \cap\left\{p_{1}, p_{2}, \ldots\right\}=\emptyset$.

As a consequence of Claim 1, we obtain that $X$ is not locally connected at $p_{0}$. Thus $p_{0} \in S$. Another consequence of Claim 1 is that $S$ cannot contain infinitely many elements of the sequence $\left\{p_{m}\right\}_{m=1}^{\infty}$.

In particular, we have that for each arc $J \subset S-\left\{z_{1}\right\}$, there exists a point $p_{0} \in S$ such that $f\left(p_{0}\right)=J$. Taking a sequence of arcs in $S-\left\{z_{1}\right\}$ converging to $S$, we obtain that there exists a point $p \in S$ such that $f(p)=S$. Let $K=f^{-1}(\{S\})$. Hence, $K \cap S \neq \emptyset$.

Since the set $F=\left\{f\left(z_{n}\right) \in C(X): n \in\{0,1,2, \ldots\}\right\}$ is countable, we can choose a point $q_{0} \in S$ such that $\left\{q_{0}\right\} \notin F$. 
For each $n \in \mathbb{N}$, fix a sequence of points $\left\{q_{m}^{(n)}\right\}_{m=1}^{\infty}$ in $R_{n}$ such that $\lim q_{m}^{(n)}=$ $q_{0}$. Since $f$ is onto, for each $m \in \mathbb{N}$, there exists a point $p_{m}^{(n)} \in X$ such that $f\left(p_{m}^{(n)}\right)=\left\{q_{m}^{(n)}\right\}$. Since $X$ is compact, we may assume that the sequence $\left\{p_{m}^{(n)}\right\}_{m=1}^{\infty}$ converges to a point $w_{n} \in X$. By the considerations we made before, $w_{n} \in S$, and we may assume that $p_{m}^{(n)} \in X-S$ for each $m \in \mathbb{N}$. If there exists $k \in \mathbb{N}$ such that $R_{k}$ contains infinitely many elements of the sequence $\left\{p_{m}^{(n)}\right\}_{m=1}^{\infty}$, we choose one $k_{n}$ with such a property and, taking a subsequence if necessary, we assume that the complete sequence $\left\{p_{m}^{(n)}\right\}_{m=1}^{\infty}$ is contained in $R_{k_{n}}$. On the other hand, if each $R_{k}$ contains finitely many elements of the sequence $\left\{p_{m}^{(n)}\right\}_{m=1}^{\infty}$, we make $k_{n}=0$.

Let $w_{0} \in S$ be a limit point of the sequence $\left\{w_{n}\right\}_{n=1}^{\infty}$. By continuity of $f$, for each $n \in \mathbb{N}, f\left(w_{n}\right)=\left\{q_{0}\right\}$. Thus $f\left(w_{0}\right)=\left\{q_{0}\right\}$. In particular, $f\left(w_{0}\right) \neq S$. Thus $w_{0} \in S-K$. Let $G$ be the component of $S-K$ which contains the point $w_{0}$.

Let $\varphi:[0,1] \rightarrow S$ be a continuous map such that $\varphi \mid(0,1):(0,1) \rightarrow G$ is a homeomorphism and $\varphi(0), \varphi(1) \in K$. By the choice of $w_{0}$, we can choose three different numbers $n, r$ and $s$ in $\mathbb{N}$ such that $w_{n}, w_{r}$ and $w_{s}$ belong to $G$. One of the two components of $G-\left\{w_{n}\right\}$ (or both) does not contain the point $z_{k_{n}}$. So one of the two intervals $\left(0, \varphi^{-1}\left(w_{n}\right)\right)$ or $\left(\varphi^{-1}\left(w_{n}\right), 1\right)$ (or both) does not intersect the set $\varphi^{-1}\left(z_{k_{n}}\right)$. The same happens with each one of the numbers $r$ and $s$. Since we have three numbers and only two choices for the intervals, we may assume that the map $\varphi$ was chosen so that the interval $\left(0, \varphi^{-1}\left(w_{n}\right)\right)$ does not intersect the set $\varphi^{-1}\left(z_{k_{n}}\right)$ and the interval $\left(0, \varphi^{-1}\left(w_{r}\right)\right)$ does not intersect the set $\varphi^{-1}\left(z_{k_{r}}\right)$. Let $b=\varphi^{-1}\left(w_{n}\right)$ and $c=\varphi^{-1}\left(w_{r}\right)$.

Let $\eta=\left\|z_{n}-z_{r}\right\|>0$. Since $\varphi(0) \in K, f(\varphi(0))=S$. Thus there exists $a \in(0, \min \{b, c\})$ such that $H(f(\varphi(a)), S)<\frac{\eta}{3}$.

Claim 2. $f(\varphi(a)) \subset S$ and $f(\varphi(a)) \in \operatorname{cl}_{C(X)}\left(C\left(R_{n}\right)\right) \cap \operatorname{cl}_{C(X)}\left(C\left(R_{r}\right)\right)$.

Note that there exists a retraction $\rho: X \rightarrow S$. Since $K=f^{-1}(\{S\})$ and $G$ is a component of $S-K$, for each $t \in[a, b], f(\varphi(t)) \neq S$. Since $S$ is a terminal subcontinuum of $X$, by the proof of Theorem 11.5 of [3], it follows that $C(S)-\{S\}$ is an arcwise component of $C(X)-\{S\}$. Since $f(\varphi([a, b])) \subset C(X)-\{S\}$ and $f(\varphi(b))=f\left(w_{n}\right)=\left\{q_{0}\right\} \in C(S)-\{S\}$, we conclude that $f(\varphi([a, b])) \subset C(S)-\{S\}$. In particular, $f(\varphi(a)) \in C(S)$. Thus, for each $t \in[a, b], \rho(f(\varphi(t)))=f(\varphi(t)) \in$ $C(S)-\{S\}$. Let $\varepsilon_{0}=\min \{H(\rho(f(\varphi(t))), S): t \in[a, b]\}>0$.

We only prove that $f(\varphi(a)) \in \mathrm{cl}_{C(X)}\left(C\left(R_{n}\right)\right)$; the proof that $f(\varphi(a)) \in$ $\operatorname{cl}_{C(X)}\left(C\left(R_{r}\right)\right)$ is similar. Let $\varepsilon>0$.

By the continuity of $\rho$ and $f$, there exists $\lambda>0$ such that, if $x, y \in X$ and $\|x-y\|<\lambda$, then $H(\rho(f(x)), \rho(f(y)))<\varepsilon_{0}$ and $H(f(x), f(y))<\varepsilon$.

Apply property (e) to the number $\lambda$ and the path $\varphi \mid[a, b]:[a, b] \rightarrow S$ to obtain a number $\delta>0$ with the above-mentioned properties.

By the choice of $n, z_{k_{n}} \notin \varphi([a, b])$. So $\varphi([a, b]) \subset S-\left\{z_{k_{n}}\right\}$. In the case that $k_{n}=0$, there exists $N \in \mathbb{N}$ such that, for each $k \geq N, z_{k} \notin \varphi([a, b])$. In this case $R_{1} \cup \ldots \cup R_{N}$ contains finitely many elements of the sequence $\left\{p_{m}^{(n)}\right\}_{m=1}^{\infty}$. Thus, we can choose $m \in \mathbb{N}$ such that $p_{m}^{(n)} \notin R_{1} \cup \ldots \cup R_{N}$ and $\left\|w_{n}-p_{m}^{(n)}\right\|<\delta$. Hence, $p_{m}^{(n)} \in R_{N_{0}}$ for some $N_{0}>N$, so $z_{N_{0}} \notin \varphi([a, b])$. In the case that $k_{n} \neq 0$, let $N_{0}=k_{n}$ and choose $m \in \mathbb{N}$ such that $\left\|w_{n}-p_{m}^{(n)}\right\|<\delta$ and $p_{m}^{(n)} \in R_{k_{n}}=R_{N_{0}}$. In any case, numbers $N_{0}$ and $m$ can be chosen in such a way that $\left\|w_{n}-p_{m}^{(n)}\right\|<\delta$, $p_{m}^{(n)} \in R_{N_{0}}$ and $z_{N_{0}} \notin \varphi([a, b])$. 
By the choice of $\delta$, there exists a path $\beta:[a, b] \rightarrow R_{N_{0}}$ such that $\beta(b)=p_{m}^{(n)}$ and $\|\varphi(t)-\beta(t)\|<\lambda$ for each $t \in[a, b]$. By the choice of $\lambda$, given $t \in[a, b]$, $H(\rho(f(\varphi(t))), \rho(f(\beta(t))))<\varepsilon_{0}$ and $H(f(\varphi(t)), f(\beta(t)))<\varepsilon$. Thus,

$$
H(f(\varphi(a)), f(\beta(a)))<\varepsilon
$$

and, by the choice of $\varepsilon_{0}, \rho(f(\beta(t))) \neq S$ for each $t \in[a, b]$.

By definition, $f(\beta(b))=f\left(p_{m}^{(n)}\right)=\left\{q_{m}^{(n)}\right\} \subset R_{n}$. We need to prove that $f(\beta(a)) \subset R_{n}$. In order to prove this inclusion, we prove that $f(\beta(t)) \subset R_{n}$ for each $t \in[a, b]$. Suppose to the contrary that there exists $t \in[a, b]$ such that $f(\beta(t)) \cap\left(X-R_{n}\right) \neq \emptyset$. Since $R_{n}$ is open in $X$, there exists $t_{0} \in[a, b]$ such that $f\left(\beta\left(t_{0}\right)\right) \cap\left(X-R_{n}\right) \neq \emptyset$ and $f(\beta(t)) \subset R_{n}$ for each $t \in\left(t_{0}, b\right]$. Note that $t_{0}<b$. Since $S \cup R_{n}$ is compact, by continuity, $f\left(\beta\left(t_{0}\right)\right) \subset S \cup R_{n}$. Thus $f\left(\beta\left(t_{0}\right)\right) \cap S \neq \emptyset$. Given $t \in\left[t_{0}, b\right]$, consider the set $E_{t}=\bigcup\left\{f(\beta(u)): u \in\left[t_{0}, t\right]\right\}$. Then $E_{t} \in C(X)$ (see [3], Lemmas 1.43 and 1.48]) and $\lim _{t \rightarrow t_{0}} E_{t}=E_{t_{0}}=f\left(\beta\left(t_{0}\right)\right)$. Note that, if $t>t_{0}$, then $f(\beta(t)) \subset E_{t}$, so $E_{t} \cap R_{n} \neq \emptyset$. Moreover, $f\left(\beta\left(t_{0}\right)\right) \subset E_{t}$, so $E_{t} \cap S \neq \emptyset$. By property (h), $S \subset E_{t}$. Taking the limit as $t \rightarrow t_{0}$, we obtain that $S \subset E_{t_{0}}=f\left(\beta\left(t_{0}\right)\right)$. Thus $S=\rho\left(f\left(\beta\left(t_{0}\right)\right)\right)$, which is a contradiction with the conclusion in the previous paragraph. Therefore, $f(\beta(a)) \subset R_{n}$.

Hence, $f(\beta(a)) \subset R_{n}$ and $H(f(\varphi(a)), f(\beta(a)))<\varepsilon$. We have shown that $f(\varphi(a)) \in \operatorname{cl}_{C(X)}\left(C\left(R_{n}\right)\right)$. This ends the proof of Claim 2.

We are ready to obtain the final contradiction. By the first part of Claim 2, $f(\varphi(a))$ is a subarc of $S$. By Claim 2 and property (f) $z_{n} \notin f(\varphi(a))$ or $z_{n}$ is an end point of $f(\varphi(a))$, and the same happens for $z_{r}$. Thus $f(\varphi(a))$ is contained in one of the two subarcs in which $S$ is divided by the two points $z_{n}$ and $z_{r}$. But this is impossible since $H(f(\varphi(a)), S)<\frac{\eta}{3}$. This completes the proof that there is no continuous map from $X$ onto $C(X)$.

\section{ACKNOWLEDGMENT}

The author wishes to thank Leonardo Espinosa for his technical help on the preparation of the final version of this paper.

\section{REFERENCES}

1. I. Kremińska and J. R. Prajs, On continua whose hyperspace of subcontinua is $\sigma$-locally connected, Topology Appl. 96 (1999), 53-61. MR.1701239 (2000e:54023)

2. S. B. Nadler, Jr., Some problems concerning hyperspaces, Topology Conference (V. P. I. and S. U.), Lecture Notes in Math., vol. 375, Springer-Verlag, New York, 1974, Raymond F. Dickman, Jr. and Peter Fletcher, editors, 190-197. MR0370465 (51:6692)

3. S. B. Nadler, Jr., Hyperspaces of Sets, Monographs and Textbooks in Pure and Applied Math. Vol. 49, Marcel Dekker, Inc., New York, 1978. MR0500811 (58:18330)

Instituto de Matematicas, UnAm, Circuito Exterior, Ciudad Universitaria, Mexico, 04510, D.F.

E-mail address: illanes@matem.unam.mx 